\title{
THE WIRELESS NETWORK JAMMING PROBLEM
}

\author{
CLAYTON W. COMMANDER, PANOS M. PARDALOS, VALERIY RYABCHENKO, STAN URYASEV, \\ AND GRIGORIY ZRAZHEVSKY
}

\begin{abstract}
In adversarial environments, disabling the communication capabilities of the enemy is a high priority. We introduce the problem of determining the optimal number and locations for a set of jamming devices in order to neutralize a wireless communication network. This problem is known as the WIRELESS NETWORK JAMMING PROBLEM. We develop several mathematical programming formulations based on covering the communication nodes and limiting the connectivity index of the nodes. Two case studies are presented comparing the formulations with the addition of various percentile constraints. Finally, directions of further research are addressed.
\end{abstract}

\section{INTRODUCTION}

Military strategists are constantly seeking ways to increase the effectiveness of their force while reducing the risk of casualties. In any adversarial environment, an important goal is always to neutralize the communication system of the enemy. In this work, we are interested in jamming a wireless communication network. Specifically, we introduce and study the problem of determining the optimal number and placement for a set of jamming devices in order to neutralize communication on the network. This is known as the WIRELESS NETWORK JAMMING PROBLEM (WNJP). Despite the enormous amount of research on optimization in telecommunications (Resende and Pardalos, 2006), this important problem for military analysts has received little attention by the research community.

The organization of the paper is as follows. Section 2 contains several formulations based on covering the communication nodes with jamming devices. In Section 3, we use tools from graph theory to define an alternative formulation based on limiting the connectivity index of the network nodes. Next, we incorporate percentile constraints to develop formulations which provide solutions requiring less jamming devices, but whose solution quality favors the exact methods. In Section 5, we present two case studies comparing the solutions and computation time for all formulations. Finally, conclusions and future directions of research are addressed.

We will now briefly introduce some of the idiosyncrasies, symbols, and notations we will employ throughout this paper. Denote a graph $G=(V, E)$ as a pair consisting of a set of vertices $V$, and a set of edges $E$. All graphs in this paper are assumed to be undirected and unweighted. We use the symbol " $b:=a$ " to mean "the expression $a$ defines the (new) symbol $b$ " in the sense of King (1994). Of course, this could be conveniently extended so that a statement like " $(1-\epsilon) / 2:=7$ " means "define the symbol $\epsilon$ so that $(1-\epsilon) / 2=7$ holds." Finally, we will use italics for emphasis and SMALL CAPS for problem names. Any other locally used terms and symbols will be defined in the sections in which they appear.

\section{Coverage Formulations}

Before formally defining the problem statement, we will state some basic assumptions about the jamming devices and the communication nodes being jammed. We assume that 
parameters such as the frequency range of the jamming devices are known. In addition, the jamming devices are assumed to have omnidirectional antennas. The communication nodes are also assumed to be outfitted with omnidirectional antennas and function as both receivers and transmitters. Given a graph $G=(V, E)$, we can represent the communication devices as the vertices of the graph. An undirected edge would connect two nodes if they are within a certain communication threshold.

Given a set $\mathcal{M}=\{1,2, \ldots, m\}$ of communication nodes to be jammed, the goal is to find a set of locations for placing jamming devices in order to suppress the functionality of the network. The jamming effectiveness of device $j$ is calculated using $d:(V \times V) \mapsto \mathbb{R}$, where $d$ is a decreasing function of the distance from the jamming device to the node being jammed. Here we are considering radio transmitting nodes, and correspondingly, jamming devices which emit electromagnetic waves. Thus the jamming effectiveness of a device depends on the power of its electromagnetic emission, which is inversely proportional to the squared distance from the jamming device to the node being jammed. Specifically,

$$
d_{i j}:=\frac{\lambda}{r^{2}(i, j)},
$$

where $\lambda \in \mathbb{R}$ is a constant, and $r(i, j)$ represents the distance between node $i$ and jamming device $j$. Without the loss of generality, we can set $\lambda=1$.

The cumulative level of jamming energy received at node $i$ is defined as

$$
Q_{i}:=\sum_{j=1}^{n} d_{i j}=\sum_{j=1}^{n} \frac{1}{r^{2}(i, j)},
$$

where $n$ is the number of jamming devices. Then, we can formulate the WIRELESS NETWORK JAMMING PROBLEM (WNJP) as the minimization of the number of jamming devices placed, subject to a set of covering constraints:

(WNJP) Minimize $n$

$$
\text { s.t. } \quad Q_{i} \geq C_{i}, \quad i=1,2, \ldots, m .
$$

The solution to this problem provides the optimal number of jamming devices needed to ensure a certain jamming threshold $C_{i}$ is met at every node $i \in \mathcal{M}$. A continuous optimization approach where one is seeking the optimal placement coordinates $\left(x_{j}, y_{j}\right), j=$ $1,2, \ldots, n$ for jamming devices given the coordinates $\left(X_{i}, Y_{i}\right), i=1,2, \ldots, m$, of network nodes, leads to highly non-convex formulations. For example, consider the covering constraint for network node $i$, which is given as

$$
\sum_{j=1}^{n} \frac{1}{\left(x_{j}-X_{i}\right)^{2}+\left(y_{j}-Y_{i}\right)^{2}} \geq C_{i} .
$$

It is easy to verify that this constraint is non-convex. Finding the optimal solution to the resulting nonlinear programming problem would require an extensive amount of computational effort.

To overcome the non-convexity of the above formulation, we propose several integer programming models for the problem. Suppose now that along with the set of communication nodes $\mathcal{M}=\{1,2, \ldots, m\}$, there is a fixed set $\mathcal{N}=\{1,2, \ldots, n\}$ of possible locations for the jamming devices. This assumption is reasonable because in real battlefield scenarios, the set of possible placement locations will likely be limited. Define the 
decision variable $x_{j}$ as

$$
x_{j}:=\left\{\begin{array}{l}
1, \text { if a jamming device is installed at location } j, \\
0, \text { otherwise. }
\end{array}\right.
$$

If we redefine $r(i, j)$ to be the distance between communication node $i$ and jamming location $j$, then we have the OPTIMAL NETWORK COVERING (ONC) formulation of the WNJP given as

$$
\begin{aligned}
\text { (ONC) Minimize } & \sum_{j=1}^{n} c_{j} x_{j} \\
\text { s.t. } & \\
& \sum_{j=1}^{n} d_{i j} x_{j} \geq C_{i}, \quad i=1,2, \ldots, m, \\
& x_{j} \in\{0,1\}, \quad j=1,2, \ldots, n,
\end{aligned}
$$

where $C_{i}$ and $d_{i j}$ are defined as above. Here the objective is to minimize the number of jamming devices used while achieving some minimum level of coverage at each node. The coefficients $c_{j}$ in (4) represent the costs of installing a jamming device at location $j$. In a battlefield scenario, placing a jamming device in the direct proximity of a network node may be theoretically possible; however, such a placement might be undesirable due to security considerations. In this case, the location considered would have a higher placement cost than would a safer location. If there are no preferences for device locations, then without the loss of generality,

$$
c_{j}=1, \quad j=1,2, \ldots, n .
$$

Though we have removed the non-convex covering constraints, this formulation remains computationally difficult. Notice that ONC is formulated as a MULTIDIMENSIONAL KNAPSACK PROBLEM which is known to be $\mathcal{N} \mathcal{P}$-hard in general (Garey and Johnson, 1979).

\section{Connectivity Formulation}

In the general WNJP, it is important that the distinction be made that the objective is not simply to jam all of the nodes, but to destroy the functionality of the underlying communication network. In this section, we use tools from graph theory to develop a method for suppressing the network by jamming those nodes with several communication links and derive an alternative formulation of the WNJP. Given a graph $G=(V, E)$, the connectivity index of a node is defined as the number of nodes reachable from that vertex (see Figure 1 for examples). To constrain the network connectivity in optimization models, we can impose constraints on the connectivity indices instead of using covering constraints.

We can now develop a formulation for the WNJP based on the connectivity index of the communication graph. We assume that the set of communication nodes $\mathcal{M}=\{1,2, \ldots, m\}$ to be jammed is known and a set of possible locations $\mathcal{N}=\{1,2, \ldots, n\}$ for the jamming devices is given. Note than in the communication graph, $V \equiv \mathcal{M}$. Let $S_{i}:=\sum_{j=1}^{n} d_{i j} x_{j}$ denote the cumulative level of jamming at node $i$. Then node $i$ is said to be jammed if $S_{i}$ exceeds some threshold value $C_{i}$. We say that communication is severed between nodes $i$ and $j$ if at least one of the nodes is jammed. Further, let $y: \mathcal{M} \times \mathcal{M} \mapsto\{0,1\}$ be a surjection where $y_{i j}:=1$ if there exists a path from node $i$ to node $j$ in the jammed network. Lastly, let $z: \mathcal{M} \mapsto\{0,1\}$ be a surjective function where $z_{i}$ returns 1 if node $i$ is not jammed. 

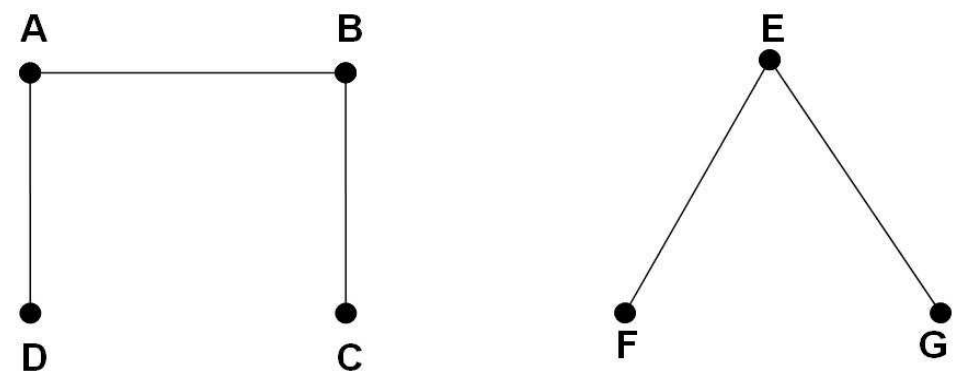

FIGURE 1. Connectivity Index of nodes A,B,C,D is 3. Connectivity Index of E,F,G is 2. Connectivity Index of $\mathrm{H}$ is 0 .

The objective of the CONNECTIVITY INDEX PROBLEM (CIP) formulation of the WNJP is to minimize the total jamming cost subject to a constraint that the connectivity index of each node does not exceed some pre-described level $L$. The corresponding optimization problem is given as:

$$
\begin{aligned}
\text { (CIP) Minimize } & \sum_{j=1}^{n} c_{j} x_{j} \\
\text { s.t. } \quad & \sum_{\substack{j=1 \\
j \neq i}}^{m} y_{i j} \leq L, \forall i \in \mathcal{M}, \\
& M\left(1-z_{i}\right)>S_{i}-C_{i} \geq-M z_{i}, \forall i \in \mathcal{M}, \\
& x_{j} \in\{0,1\}, \forall j \in \mathcal{N}, \\
& z_{i} \in\{0,1\} \forall i \in \mathcal{M}, \\
& \forall i, j \in \mathcal{M}, y_{i j} \in\{0,1\}, \forall i, j \in \mathcal{M},
\end{aligned}
$$

where $M \in \mathbb{R}$ is some large constant.

Let $v: \mathcal{M} \times \mathcal{M} \mapsto\{0,1\}$ and $v^{\prime}: \mathcal{M} \times \mathcal{M} \mapsto\{0,1\}$ be defined as follows:

$$
v_{i j}:=\left\{\begin{array}{l}
1, \text { if }(i, j) \in E \\
0, \text { otherwise }
\end{array}\right.
$$

and

$$
v_{i j}^{\prime}:=\left\{\begin{array}{l}
1, \text { if }(i, j) \text { exists in the jammed network } \\
0, \text { otherwise. }
\end{array}\right.
$$


With this, we can formulate an equivalent integer program as

$$
\begin{aligned}
\text { (CIP-1) Minimize } & \sum_{j=1}^{n} c_{j} x_{j}, \\
\text { s.t. } \quad & y_{i j} \geq v_{i j}^{\prime}, \forall i, j \in \mathcal{M}, \\
& y_{i j} \geq y_{i k} y_{k j}, k \neq i, j ; \forall i, j \in \mathcal{M}, \\
& v_{i j}^{\prime} \geq v_{i j} z_{j} z_{i}, i \neq j ; \forall i, j \in \mathcal{M}, \\
& \sum_{j=1}^{m} y_{i j} \leq L, \forall i \in \mathcal{M}, \\
& j_{j \neq i} \\
M\left(1-z_{i}\right) \quad>\quad & S_{i}-C_{i} \geq-M z_{i}, \forall i \in \mathcal{M}, \\
& z_{i} \in\{0,1\}, \forall i \in \mathcal{M}, \\
& y_{i j} \in\{0,1\} \forall i, j \in \mathcal{M}, \\
& v_{i j}^{\prime} \in\{0,1\}, \forall i, j \in \mathcal{M} .
\end{aligned}
$$

Lemma 1. If CIP has an optimal solution then, CIP-1 has an optimal solution. Further, any optimal solution $x^{*}$ of the optimization problem CIP-1 is an optimal solution of CIP.

Proof. It is easy to establish that if $i$ and $j$ are reachable from each other in the jammed network then in CIP- $1, y_{i j}=1$. Indeed, if $i$ and $j$ are adjacent then there exists a sequence of pairwise adjacent vertices:

$$
\left\{\left(i_{0}, i_{1}\right), \ldots,\left(i_{m-1}, i_{m}\right)\right\},
$$

where $i_{0}=i$, and $i_{m}=j$. Using induction it can be shown that $y_{i_{0} i_{k}}=1, \forall k=$ $1,2, \ldots, m$. From (16), we have that $y_{i_{k} i_{k+1}}=1$. If $y_{i_{0} i_{k}}=1$, then by (17), $y_{i_{0} i_{k+1}} \geq$ $y_{i_{0} i_{k}} y_{i_{k} i_{k+1}}=1$, which proves the induction step.

The proven property implies that in CIP-1:

$$
\sum_{\substack{j=1 \\ j \neq i}}^{m} y_{i j} \geq \text { connectivity index of } i \text {. }
$$

Therefore, if $\left(x^{*}, y^{*}\right)$ and $\left(x^{* *}, y^{* *}\right)$ are optimal solutions of CIP-1 and CIP correspondingly, then:

$$
V\left(x^{*}\right) \geq V\left(x^{* *}\right),
$$

where $V$ is the objective in CIP- 1 and CIP.

As $\left(x^{* *}, y^{* *}\right)$ is feasible in CIP, it can be easily checked that $y^{* *}$ satisfies all feasibility constraints in CIP-1 (it follows from the definition of $y_{i j}$ in CIP). So, $\left(x^{* *}, y^{* *}\right)$ is feasible in CIP-1; thus proving the first statement of the lemma.

Hence from CIP-1,

$$
V\left(x^{* *}\right) \geq V\left(x^{*}\right) .
$$

From (26) and (27):

$$
V\left(x^{* *}\right)=V\left(x^{*}\right) .
$$

Let us define $y$ such that

$$
y_{i j}:=1 \Leftrightarrow j \text { is reachable from } i \text { in the network jammed by } x^{*} .
$$


Using (25), $\left(x^{*}, y\right)$ is feasible in CIP-1, and hence optimal. From the construction of $y$ it follows that $\left(x^{*}, y\right)$ is feasible in CIP. Relying on (28) we can claim that $x^{*}$ is an optimal solution of CIP. The lemma is proved.

We have therefore established a one-to-one correspondence between formulations CIP and CIP-1. Now, we can linearize the integer program CIP-1 by applying some standard transformations. The resulting linear 0-1 program, CIP-2 is given as

$$
\begin{aligned}
\text { (CIP-2) Minimize } & \sum_{j=1}^{n} c_{j} x_{j} \\
\text { s.t. } & \\
& y_{i j} \geq v_{i j}^{\prime}, \forall i, j=1, \ldots, \mathcal{M}, \\
& y_{i j} \geq y_{i k}+y_{k j}-1, k \neq i, j ; \forall i, j \in \mathcal{M}, \\
& v_{i j}^{\prime} \geq v_{i j}+z_{j}+z_{i}-2, i \neq j ; \forall i, j \in \mathcal{M}, \\
& \sum_{j=1}^{m} y_{i j} \leq L, \forall i \in \mathcal{M}, \\
& j \neq i \\
& M\left(1-z_{i}\right)>S_{i}-C_{i} \geq-M z_{i}, \forall i \in \mathcal{M}, \\
& z_{i} \in\{0,1\}, \forall i \in \mathcal{M}, \\
& y_{i j} \in\{0,1\} \forall i, j \in \mathcal{M}, \\
& v_{i j}^{\prime} \in\{0,1\}, \forall i, j \in \mathcal{M} .
\end{aligned}
$$

In the following lemma, we provide a proof of equivalence between CIP- 1 and CIP- 2 .

Lemma 2. If CIP-1 has an optimal solution then CIP-2 has an optimal solution. Furthermore, any optimal solution $x^{*}$ of CIP-2 is an optimal solution of CIP- 1 .

Proof. For 0-1 variables the following equivalence holds:

$$
y_{i j} \geq y_{i k} y_{k j} \Leftrightarrow y_{i j} \geq y_{i k}+y_{k j}-1
$$

The only differences between CIP- 1 and CIP- 2 are the constraints:

$$
\begin{array}{r}
v_{i j}^{\prime}=v_{i j} z_{j} z_{i} \\
v_{i j}^{\prime} \geq v_{i j}+z_{i}+z_{j}-2
\end{array}
$$

Note that (38) implies (39) $\left(v_{i j} z_{j} z_{i} \geq v_{i j}+z_{i}+z_{j}-2\right)$. Therefore, the feasibility region of CIP-2 includes the feasibility region of CIP-1. This proves the first statement of the lemma.

From the last property we can also deduce that for all $x_{1}, x_{2}$ such that $x_{1}$ is an optimal solution of CIP- 1 , and $x_{2}$ is optimal for CIP-2, that

$$
V\left(x_{1}\right) \geq V\left(x_{2}\right)
$$

where $V(x)$ is the objective of CIP-1 and CIP-2.

Let $\left(x^{*}, y^{*}, v^{\prime *}, z^{*}\right)$ be an optimal solution of CIP-2. Construct $v^{\prime \prime}$ using the following rules:

$$
v_{i j}^{\prime \prime}:=\left\{\begin{array}{l}
1, \text { if } v_{i j}+z_{i}^{*}+z_{j}^{*}-2=1 \\
0, \text { otherwise. }
\end{array}\right.
$$


$v_{i j}^{\prime *} \geq v_{i j}^{\prime \prime *} \Rightarrow\left(x^{*}, y^{*}, v^{\prime \prime *}, z^{*}\right)$ is feasible in CIP-2 $\left(y_{i j} \geq v_{i j}^{\prime \prime}\right)$, hence optimal (the objective value is $V\left(x^{*}\right)$, which is optimal). Using (41), $\left(v^{\prime \prime}, z^{*}\right)$ satisfies:

$$
v_{i j}^{\prime \prime}=v_{i j} z_{j}^{*} z_{i}^{*} .
$$

Using this we have that $\left(x^{*}, y^{*}, v^{\prime \prime} *, z^{*}\right)$ is feasible for CIP-1. If $x_{1}$ is an optimal solution of CIP-1 then:

$$
V\left(x_{1}\right) \leq V\left(x^{*}\right)
$$

On the other hand, using (40):

$$
V\left(x^{*}\right) \leq V\left(x_{1}\right)
$$

(42) and (43) together imply $V\left(x_{1}\right)=V\left(x^{*}\right)$. The last equality proves that $x^{*}$ is an optimal solution of CIP-1. Thus, the lemma is proved.

We have as a result of the above lemmata the following theorem which states that the optimal solution to the linearized integer program CIP-2 is an optimal solution to the original connectivity index problem CIP.

Theorem 1. If CIP has an optimal solution then CIP-2 has an optimal solution. Furthermore, any optimal solution of CIP-2 is an optimal solution of CIP.

Proof. The theorem is an immediate corollary of Lemma 1 and Lemma 2.

\section{Deterministic Setup with Percentile Constraints}

As we have seen, to suppress communication on a wireless network may not necessarily imply that all nodes must be jammed. We might instead choose to constrain the connectivity index of the nodes as in the CIP formulations. Alternatively, it may be sufficient to jam some percentage of the total number of nodes in order to acquire an effective control over the network. The latter can be accomplished by adding percentile risk constraints to the mathematical formulation. Used extensively in financial engineering applications and optimization of stochastic systems, risk measures have also proven effective when applied to deterministic problems (Krokhmal, Murphey, Pardalos, Uryasev and Zrazhevski, 2003). In this section, we review two risk measures, namely Value-at-Risk (VaR) and Conditional Value-at-Risk $(\mathrm{CVaR})$ and provide formulations of the WNJP with the incorporation of these risk measures.

4.1. Value-at-Risk (VaR) and Conditional Value-at-Risk (CVaR). The Value-at-Risk $(\mathrm{VaR})$ percentile measure is perhaps the most widely used in all applications of risk management (Holton, 2003). Stated simply, VaR is an upper percentile of a given loss distribution. In other words, given a specified confidence level $\alpha$, the corresponding $\alpha$-VaR is the lowest amount $\zeta$ such that, with probability $\alpha$, the loss is less or equal to $\zeta$ (Krokhmal, Palmquist and Uryasev, 2002). VaR type risk measures are popular for several reasons including their simple definition and ease of implementation.

An alternative risk measure is Conditional Value-at-Risk (CVaR). Developed by Rockafellar and Uryasev, $\mathrm{CVaR}$ is a percentile risk measure constructed for estimation and control of risks in stochastic and uncertain environments. However, CVaR-based optimization techniques can also be applied in a deterministic percentile framework. CVaR is defined as the conditional expected loss under the condition that it exceeds VaR (Uryasev, 2000). Figure 2 provides a graphical representation of the VaR and CVaR concepts. As we will see, CVaR has many properties that offer nice alternatives to VaR. 


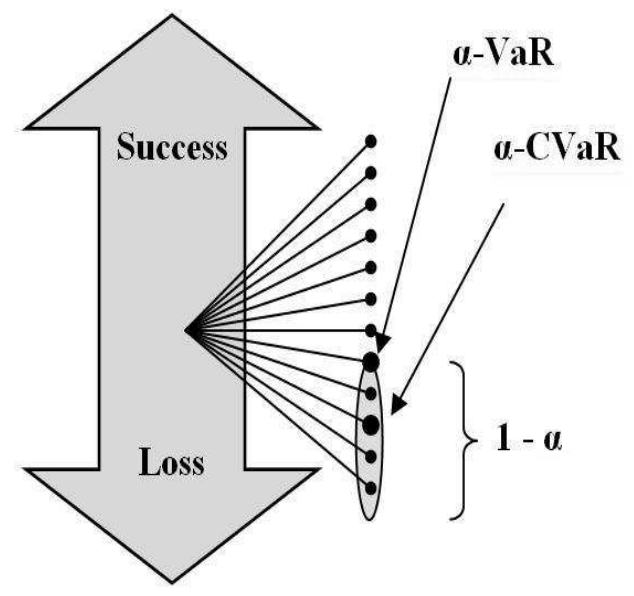

FIGURE 2. Graphical representation of Var and CVaR.

Let $f(x, y)$ be a performance or loss function associated with the decision vector $x \subseteq$ $X \subseteq \mathbb{R}^{n}$, and a random vector in $y \in \mathbb{R}^{m}$. The $y$ vector can be interpreted as the uncertainties that may affect the loss. Then, for each $x \in X$, the corresponding loss $f(x, y)$ is a random variable having a distribution in $\mathbb{R}$ which is induced by $y$. We assume that $y$ is governed by a probability measure $P$ on a Borel set, say $Y$. Therefore, the probability of $f(x, y)$ not exceeding some threshold value $\zeta$ is given by

$$
\psi(x, \zeta):=P\{y \mid f(x, y) \leq \zeta\} .
$$

For a fixed decision vector $x, \psi(x, \zeta)$ is the cumulative distribution function of the loss associated with $x$. This function is fundamental for defining VaR and CVaR (Krokhmal, Palmquist and Uryasev, 2002).

With this, the $\alpha$-VaR and $\alpha$-CVaR values for the loss random variable $f(x, y)$ for any specified $\alpha \in(0,1)$ are denoted by $\zeta_{\alpha}(x)$ and $\phi_{\alpha}(x)$ respectively. From the aforementioned definitions, they are given by

$$
\zeta_{\alpha}(x):=\min \{\zeta \in \mathbb{R}: \psi(x, \zeta) \geq \alpha\},
$$

and

$$
\phi_{\alpha}(x):=E\left\{f(x, y) \mid f(x, y) \geq \zeta_{\alpha}(x)\right\} .
$$

Notice that the probability that $f(x, y) \geq \zeta_{\alpha}(x)$ is equal to $1-\alpha$. Finally by definition, we have that $\phi_{\alpha}(x)$ is the conditional expectation that the loss corresponding to $x$ is greater than or equal to $\zeta_{a}(x)$ (Rockafellar and Uryasev, 2000).

The key to including $\mathrm{VaR}$ and $\mathrm{CVaR}$ constraints into a model are the characterizations of $\zeta_{\alpha}(x)$ and $\phi_{\alpha}(x)$ in terms of a function $F_{\alpha}: X \times \mathbb{R} \mapsto \mathbb{R}$ defined by

$$
F_{\alpha}(x, \zeta):=\zeta+\frac{1}{(1-\alpha)} E\{\max \{f(x, y)-\zeta, 0\}\} .
$$

The following theorem, which provides the crucial properties of the function $F_{\alpha}$ follow directly from the paper by Rockafellar and Uryasev (2000). 
Theorem 2. As a function of $\zeta, F_{\alpha}(x, \zeta)$ is convex and continuously differentiable. The $\alpha-C V a R$ of the loss associated with any $x \in X$ can be determined from the formula

$$
\phi_{\alpha}(x)=\min _{\zeta \in \mathbb{R}} F_{\alpha}(x, \zeta) \text {. }
$$

In this formula, the set consisting of the values of $\zeta$ for with the minimum is attained, namely

$$
A_{\alpha}(x)=\underset{\zeta \in \mathbb{R}}{\operatorname{argmin}} F_{\alpha}(x, \zeta),
$$

is a nonempty, closed, bounded interval, and the $\alpha$-VaR of the loss is given by

$$
\zeta_{\alpha}(x)=\text { left endpoint of } A_{\alpha}(x) \text {. }
$$

In particular, it is always the case that

$$
\zeta_{\alpha}(x) \in \underset{\zeta \in \mathbb{R}}{\operatorname{argmin}} F_{\alpha}(x, \zeta) \quad \text { and } \quad \psi_{\alpha}(x)=F_{\alpha}\left(x, \zeta_{\alpha}(x)\right) .
$$

This result provides an efficient linear optimization algorithm for CVaR. However, from a numerical perspective, the convexity of $F_{\alpha}(x, \zeta)$ with respect to $x$ and $\zeta$ as provided by Theorem 2 is more valuable than the convexity of $\phi_{\alpha}(x)$ with respect to $x$. As we will see in the following theorem due to Rockafellar and Uryasev (2002), this allows us to minimize CVaR without having to proceed numerically through repeated calculations of $\phi_{\alpha}(x)$ for various decisions $x$.

Theorem 3. Minimizing $\phi_{\alpha}(x)$ with respect to $x \in X$ is equivalent to minimizing $F_{\alpha}(x, \zeta)$ over all $(x, \zeta) \in X \times \mathbb{R}$, in the sense that

$$
\min _{x \in X} \phi_{\alpha}(x)=\min _{(x, \zeta) \in X \times \mathbb{R}} F_{\alpha}(x, \zeta),
$$

where moreover

$$
\left(x^{*}, \zeta^{*}\right) \in \underset{(x, \zeta) \in X \times \mathbb{R}}{\operatorname{argmin}} F_{\alpha}(x, \zeta) \Leftrightarrow x^{*} \in \underset{x \in X}{\operatorname{argmin}} \phi_{\alpha}(x), \zeta^{*} \in \underset{\zeta \in \mathbb{R}}{\operatorname{argmin}} F_{\alpha}\left(x^{*}, \zeta\right) .
$$

In the deterministic setting of the WNJP, we are not particularly interested in minimizing $\mathrm{VaR}$ or $\mathrm{CVaR}$ as it pertains to the loss. Rather, we would like to impose percentile constraints on the optimization model in order to handle a desired probability threshold. The following theorem from Rockafellar and Uryasev (2002) provides this capability.

Theorem 4. For any selection of probability thresholds $\alpha_{i}$ and loss tolerances $\omega_{i}, i=$ $1, \ldots, m$, the problem

$$
\begin{array}{rl}
\min _{x \in X} & g(x) \\
\text { s.t. } & \\
& \phi_{\alpha_{i}}(x) \leq \omega_{i}, \text { for } i=1, \ldots, m,
\end{array}
$$

where $g$ is any objective function defined on $X$, is equivalent to the problem

$$
\begin{array}{rl}
\min _{\left(x, \zeta_{1}, \ldots, \zeta_{m}\right) \in X \times \mathbb{R}^{m}} & g(x) \\
\text { s.t. } & \\
& F_{\alpha_{i}}\left(x, \zeta_{i}\right) \leq \omega_{i}, \text { for } i=1, \ldots, m .
\end{array}
$$

Indeed, $\left(x^{*}, \zeta_{1}^{*}, \ldots, \zeta_{m}^{*}\right)$ solves the second problem if and only if $x^{*}$ solves the first problem and the inequality $F_{\alpha_{i}}\left(x, \zeta_{i}\right) \leq \omega_{i}$ holds for $i=1, \ldots, m$.

Furthermore, $\phi_{\alpha_{i}}\left(x^{*}\right) \leq \omega_{i}$ holds for all $i=1, \ldots, m$. In particular, for each $i$ such that $F_{\alpha_{i}}\left(x^{*}, \zeta^{*}\right)=\omega_{i}$, one has that $\phi_{\alpha_{i}}\left(x^{*}\right)=\omega_{i}$. 
4.2. Percentile Constraints and the WNJP. In this section, we investigate the use of VaR and CVaR constraints when applied to the formulations of the WNJP derived in Sections 2 and 3 above. As we have seen, risk measures are generally designed for optimization under uncertainty. Since we are considering deterministic formulations of the WNJP, we can interpret each communication node $i \in \mathcal{M}$ as a random scenario, and apply the desired risk measures in this context.

We begin with the OPTIMAL NETWORK COVERING formulation of the WNJP. Suppose it is determined that jamming some fraction $\alpha \in(0,1)$ of the nodes is sufficient for effectively dismantling the network. This can be accomplished by the inclusion of $\alpha$-VaR constraints in the original model. Let $y: \mathcal{M} \mapsto\{0,1\}$ be a surjection defined by

$$
y_{i}:=\left\{\begin{array}{l}
1, \text { if node } i \text { is jammed } \\
0, \text { otherwise. }
\end{array}\right.
$$

Recall from Section 2 that $\mathcal{N}=\{1, \ldots, n\}$ is the set of locations for the jamming devices, and $x$ is a binary vector of length $n$ where $x_{j}=1$ if a jamming device is placed at location $j$. Then to find the minimum number of jamming devices that will allow for covering $\alpha \cdot 100 \%$ of the network nodes with prescribed levels of jamming $C_{i}$, we must solve the following integer program

$$
\begin{aligned}
\text { (ONC-VaR) Minimize } & \sum_{j=1}^{n} c_{j} x_{j} \\
\text { s.t. } \quad & \sum_{i=1}^{m} y_{i} \geq \alpha m, \\
& \sum_{j=1}^{n} d_{i j} x_{j} \geq C_{i} y_{i}, \quad i=1,2, \ldots, m, \quad j=1,2, \ldots, n, \\
& x_{j} \in\{0,1\}, \quad j=1,2, \ldots, m . \\
& y_{i} \in\{0,1\}, \quad i=1,2,
\end{aligned}
$$

Notice that this formulation differs from the ONC formulation with the addition of the $\alpha$ VaR constraint (60). According to (61), if $y_{i}=1$ then node $i$ is jammed. Lastly, we have from (60) that at least $100 \cdot \alpha \%$ of the $y$ variables are equal to 1 .

The optimal solution to the ONC-VaR formulation will provide the minimum number of jamming devices required to suppress communication on at least $\alpha \cdot 100 \%$ of the network nodes. The resulting solution may provide coverage levels comparable to those provided by the ONC model, while potentially reducing the number of jamming devices used. However, notice that the remaining $(1-\alpha) \cdot 100 \%$ of the nodes for which $y_{i}$ is potentially 0 , there is no guarantee that they will receive any amount of coverage. Furthermore, the addition of the $m$ binary variables adds a computational burden to a problem which is already $\mathcal{N} \mathcal{P}$-hard.

We can also reformulate the CONNECTIVITY INDEX PROBLEM to include Value-atRisk constraints. Let $\rho: \mathcal{M} \mapsto \mathbb{Z}^{+}$be a surjection where $\rho_{i}$ returns the connectivity index of node $i$. That is, $\rho_{i}:=\sum_{j=1, j \neq i}^{m} y_{i j}$. Further let $w: \mathcal{M} \mapsto\{0,1\}$ be a decision variable having the property that if $w_{i}=1$, then $\rho_{i} \leq L$. With this, the connectivity index 
formulation of WNJP with VaR percentile constraints is given as

$$
\begin{aligned}
\text { (CIP-VaR) Minimize } & \sum_{j=1}^{n} c_{j} x_{j} \\
\text { s.t. } & \rho_{i} \leq L w_{i}+\left(1-w_{i}\right) M, i=1,2, \ldots, m, \\
& \sum_{i=1}^{m} w_{i} \geq \alpha m, \\
& x_{j} \in\{0,1\}, j=1,2, \ldots, n, \\
& w_{i} \in\{0,1\}, i=1,2, \ldots, m, \\
& \rho_{i} \in\{0,1\}, i=1,2, \ldots, m,
\end{aligned}
$$

where $M \in \mathbb{R}$ is some large constant.

Analogous to constraints (60)-(61), constraints (65)-(66) guarantee that at least $\alpha \cdot 100 \%$ of the nodes will have connectivity index less than $L$. As with the ONC-VaR formulation, there are two drawbacks of CIP-VaR. First, there is no control guarantee at all on any of the remaining $(1-\alpha) \cdot 100 \%$ nodes for which $w_{i}=0$. Secondly, the addition of $m$ binary variables adds a tremendous computational burden to the problem. As an alternative to VaR, we now examine formulations of the WNJP using Conditional Value-at-Risk constraints (Rockafellar and Uryasev, 2000).

We first consider the OPTIMAL NETWORK COVERING problem. In order to put this into our derived framework, we need to define the loss function associated with an instance of the ONC. We introduce the function $f:\{0,1\}^{n} \times \mathcal{M} \mapsto \mathbb{R}$ defined by

$$
f(x, i):=C_{i}-\sum_{j=1}^{n} x_{j} d_{i j}
$$

That is, given a decision vector $x$ representing the placement of the jamming devices, the loss function is defined as the difference between the energy required to jam the network node $i$ and the cumulative amount of energy received at node $i$ due to $x$. With this, we can formulate the $\mathrm{ONC}$ with the addition of $\mathrm{CVaR}$ constraints as the following integer linear program:

$$
\text { (ONC-CVaR) Minimize } \sum_{j=1}^{n} c_{j} x_{j}
$$

s.t.

$$
\begin{aligned}
& \zeta+\frac{1}{(1-\alpha) m} \sum_{i=1}^{m} \max \left\{C_{\min }-\sum_{j=1}^{n} x_{j} d_{i j}-\zeta, 0\right\} \leq 0, \\
& \zeta \in \mathbb{R}, \\
& x_{j} \in\{0,1\},
\end{aligned}
$$

where $C_{\min }$ is the minimal prescribed jamming level and $d_{i j}$ is defined as above. The expression on the left hand side of (72) is $F_{\alpha}(x, \zeta)$. Further, from Theorem 4 we see that constraint (72) corresponds to having $\phi_{\alpha}(x) \leq \omega=0$ (Rockafellar and Uryasev, 2002). Said differently, the CVaR constraint (72) implies that in the $(1-\alpha) \cdot 100 \%$ of the worst (least) covered nodes, the average value of $f(x) \leq 0$. For the case when $C_{i} \equiv C$ for all $i$, it follows that the average level of jamming energy received by the worst $(1-\alpha) \cdot 100 \%$ of nodes exceeds $C$. 
The important point about this formulation is that we have not introduced additional integer variables to the problem in order to add the percentile constraints. Recall, that in ONC-VaR we introduced $m$ discrete variables. Since we have to add only $m$ real variables to replace max-expressions under the summation and a real variable $\zeta$, this formulation is much easier to solve than ONC-VaR.

In a similar manner, we can formulate the CONNECTIVITY INDEX PROBLEM with the addition of $\mathrm{CVaR}$ constraints. As before, we need to first define an appropriate loss function. Recall that the definition of $\rho_{i}$, the connectivity index of node $i$, is given as the number of nodes reachable from $i$. Then can define the loss function $f^{\prime}$ for a network node $i$ as the difference between the connectivity index of $i$ and the maximum allowable connectivity index $L$ which occurs as a result of the placement of the jamming devices according to $x$. That is, let $f^{\prime}:\{0,1\}^{n} \times \mathcal{M} \mapsto \mathbb{Z}$ be defined by

$$
f^{\prime}(x, i):=\rho_{i}-L \text {. }
$$

With this, the CIP-CVaR formulation is given as follows.

$$
\begin{aligned}
& \text { (CIP-CVaR) Minimize } \sum_{j=1}^{n} c_{j} x_{j} \\
& \text { s.t. } \\
& \zeta+\frac{1}{(1-\alpha) m} \sum_{i=1}^{m} \max \left\{\rho_{i}-L-\zeta, 0\right\} \leq 0, \\
& \rho_{i} \in \mathbb{Z}, \\
& \zeta \in \mathbb{R},
\end{aligned}
$$

where $\rho_{i}$ is defined as above. As with the previous formulation, the expression on the lefthand side of (77) is $F_{\alpha}(x, \zeta)$ from (47). Furthermore, we have from from Theorem 4 that (77) corresponds to having $\phi_{\alpha}(x) \leq \omega=0$. This constraint on CVaR provides that for the $(1-\alpha) \cdot 100 \%$ of the worst cases, the average connectivity index will not exceed $L$. Again, we see that in order to include the CVaR constraint, we only need to add $(m+1)$ real variables to the problem. Computationally, CVaR provides a more conservative solution and will be much easier to solve than the CIP-VaR formulation as we will see in the next section.

\section{Case Studies}

In order to demonstrate the advantages and disadvantages of the proposed formulations for the WNJP, we will present two case studies. The experiments were performed on a PC equipped with a $1.4 \mathrm{MHz}$ Intel Pentium ${ }^{\circledR} 4$ processor with $1 \mathrm{~GB}$ of RAM, working under the Microsoft Windows ${ }^{\circledR}$ XP SP1 operating system. In the first study, an example network is given and the problem is modeled using the proposed coverage formulation. The problem is then solved exactly using the commercial integer programming software package, CPLEX ${ }^{\circledR}$. Next, we modify the problem to include VaR and CVaR constraints and again use CPLEX ${ }^{\circledR}$ to solve the resulting problems. Numerical results are presented and the three formulations are compared. In the second case study, we model and solve the problem using the connectivity index formulation. We then include percentile constraints re-optimize. Finally, we analyze the results.

5.1. Coverage Formulation. Here we present two networks and solve the WNJP using the network covering (ONC) formulation. The first network has 100 communication nodes and the number of available jamming devices is 36 . The cost of placing a jamming device 


\begin{tabular}{|c||c||l|}
\hline Optimal Solutions & Regular Constraints & VaR Constraints \\
\hline Number of Jammers & 6 & 4 \\
\hline Level of Jamming & $100 \% \forall$ nodes & $100 \%$ for $96 \%$ of nodes, \\
& & $85 \%$ (of reqd.) for $4 \%$ of nodes \\
\hline CPLEX $\left.^{(}\right)$Time & $0.81 \mathrm{sec}$ & $0.98 \mathrm{sec}$ \\
\hline
\end{tabular}

TABLE 1. Optimal solutions using the coverage formulation with regular and VaR constraints.

at location $j, c_{j}$ is equal to 1 for all locations. This problem was solved using the regular constraints and the VaR type constraints. Recall that there is a set of possible locations at which jamming devices can be placed. In these examples, this set of points constitutes a uniform grid over the battlespace. The placement of the jamming devices from each solution can be seen in Figure 3. The numerical results detailing the level of jamming for the network nodes is given in Table 1. Notice that the VaR solution called for 33\% less jamming devices than the original problem while providing almost the same jamming quality.

In the second example, the network has 100 communication nodes and 72 available jammers. This problem was solved using the regular constraints as well as both types of percentile constraints. The resulting graph is shown in Figure 4. The corresponding numerical results are given in Table 2.

In this example, the $\mathrm{VaR}$ formulation requires $11 \%$ less jamming devices with almost the same quality as the formulation with the standard constraints. However, this formulation requires nearly 16 hours of computation time. The $\mathrm{CVaR}$ formulation gives a solution

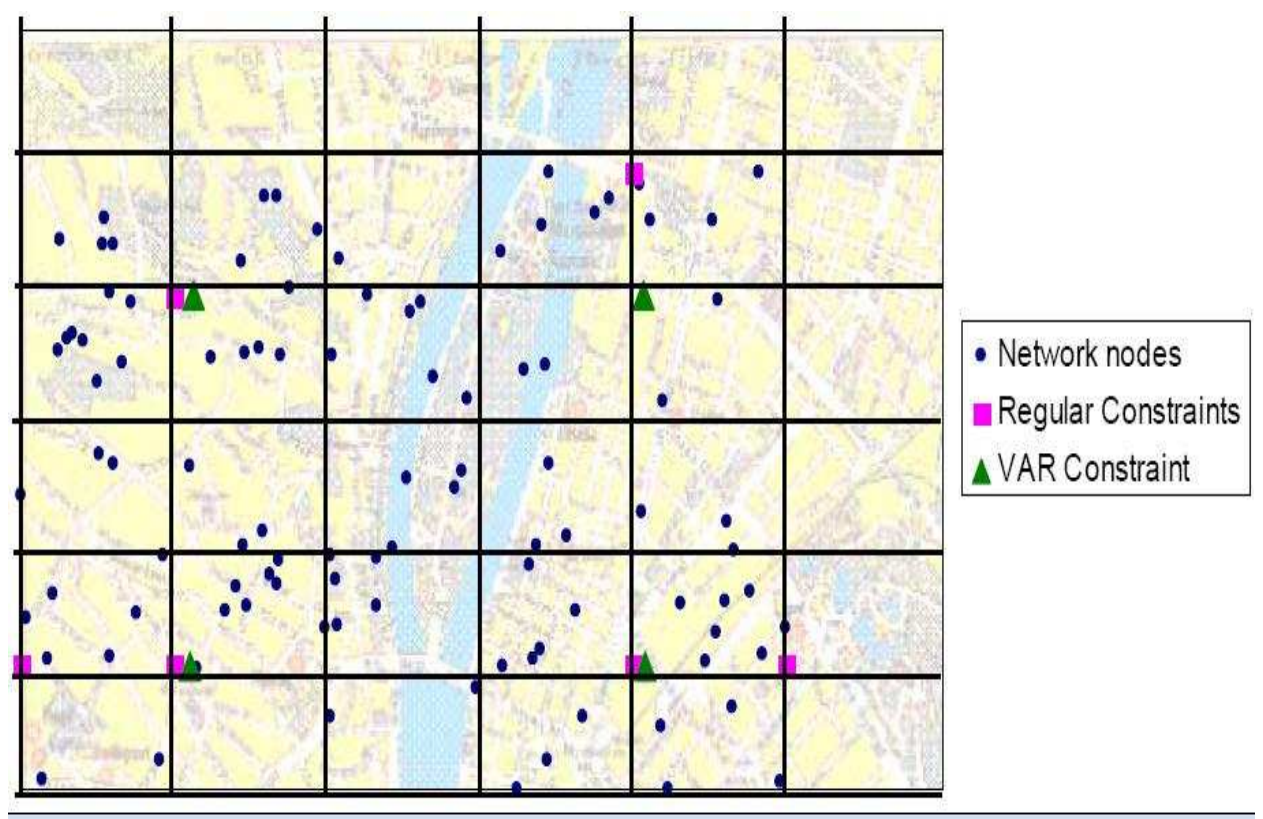

FIgURE 3. Case study 1. The placement of jammers is shown when the problem is solved using the original and VaR constraints. 


\begin{tabular}{|c|c|c|c|}
\hline Opt Solns & Reg (all) & VaR $(.9$ conf $)$ & CVaR (.7 conf) \\
\hline \# Jammers & 9 & 8 & 7 \\
\hline Jamming Level & $100 \% \forall$ nodes & $\begin{array}{l}100 \% \text { for } 90 \% \text { of } \\
\text { nodes, } \\
72 \% \text { for } 10 \% \text { of } \\
\text { nodes }\end{array}$ & $\begin{array}{l}100 \% \text { for } 57 \% \text { of } \\
\text { nodes, } \\
90 \% \text { for } 20 \% \text { of } \\
\text { nodes, } \\
76 \% \text { for } 23 \% \text { of } \\
\text { nodes }\end{array}$ \\
\hline CPLEX $^{(R)}$ Time & $15 \mathrm{sec}$ & $15 \mathrm{~h} 55 \mathrm{~min} 11 \mathrm{sec}$ & $41 \mathrm{sec}$ \\
\hline
\end{tabular}

TABLE 2. Optimal solutions using the coverage formulation with regular and VaR, and CVaR constraints.

with a very good jamming quality and requires $22 \%$ less jamming devices than the standard formulation and $11 \%$ less devices than the VaR formulation. Furthermore, the CVaR formulation requires an order of magnitude less computing time than the formulation with VaR constraints.

5.2. Connectivity Formulation. We now present a case study where the WNJP was solved using the connectivity index formulation (CIP). The communication graph consists of 30 nodes and 60 edges. The maximal number of jamming devices available is 36 . We set the maximal allowed connectivity index of any node to be 3. In Figure 5 we can see the original graph with the communication links prior to jamming. The result of the VaR and CVaR solutions is seen in Figure 6. The confidence level for both the VaR and CVaR formulations was 0.9 . Both formulations provide optimal solutions for the given instance.

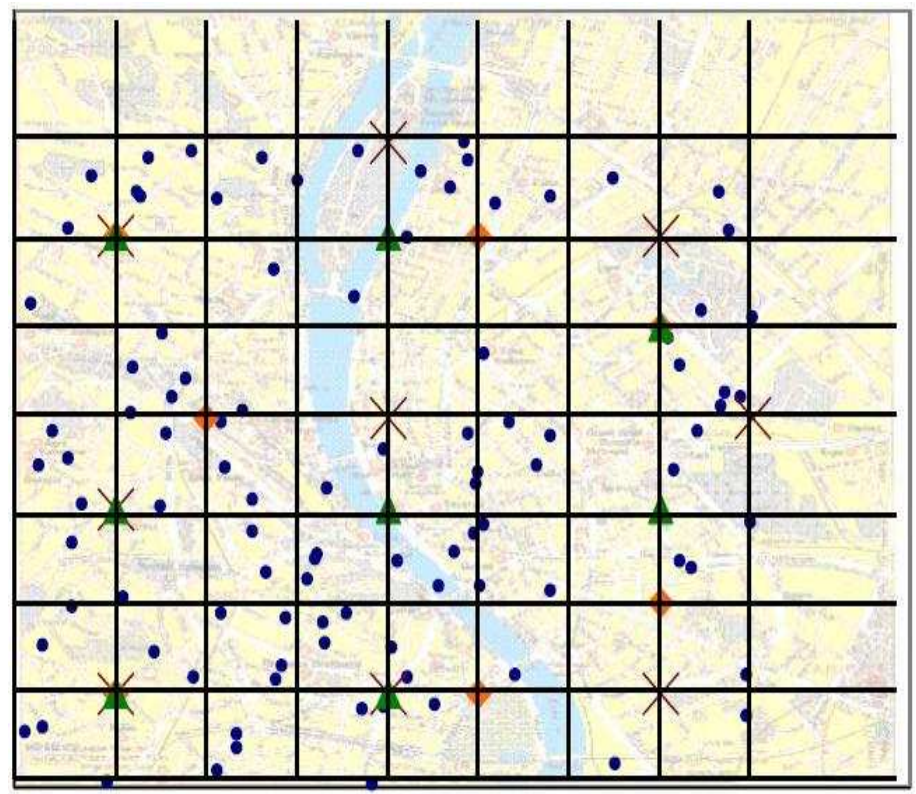

- Network Nodes $\times$ Regular Constraints - CVAR Constraint $\triangle$ VAR Constraint

FIGURE 4. Case study 1 continued. The placement of jammers is shown when the problem is solved using VaR and CVaR constraints. 


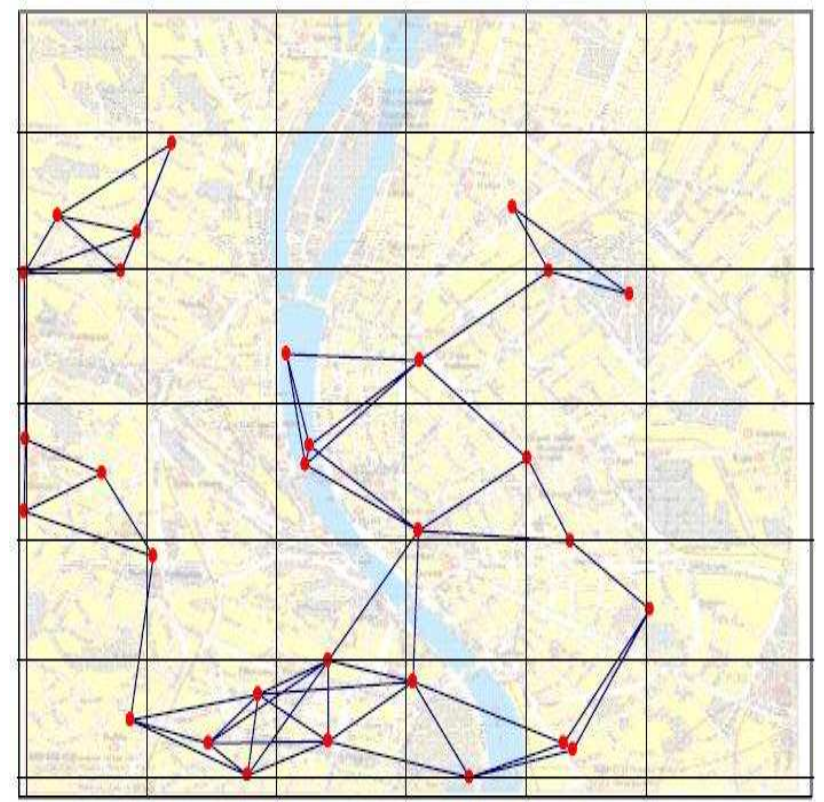

FIGURE 5. Case Study 2: Original graph.

The resulting computation time for the VaR formulation was 15 minutes 34 seconds, while the CVaR formulation required only 7 minutes 33 seconds.

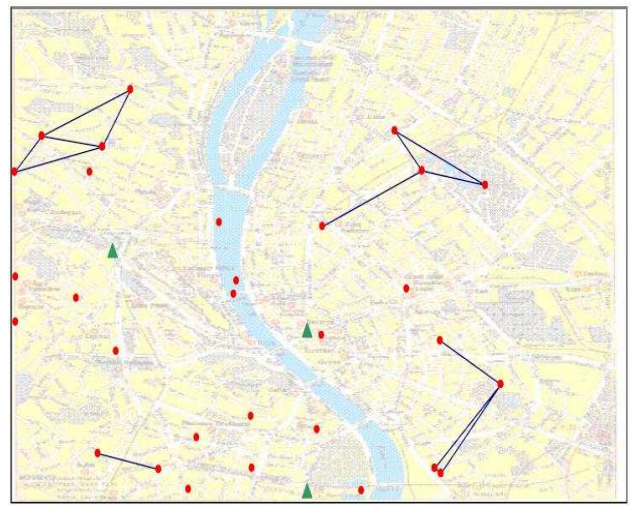

(a)

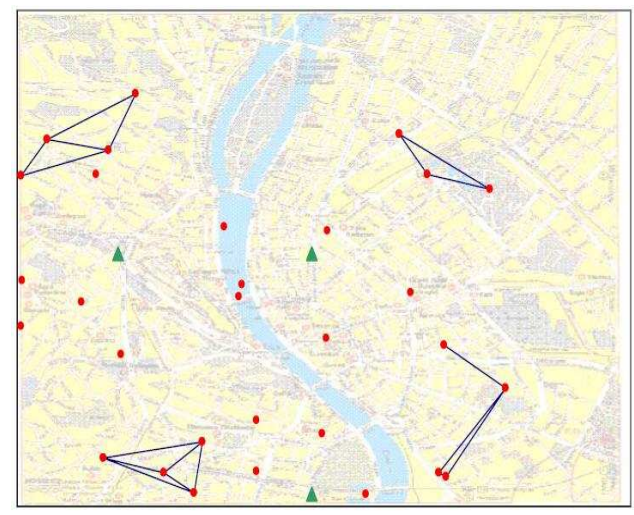

(b)

FIgURE 6. (a) VaR Solution. (b) CVaR Solution. In both cases, the triangles represent the jammer locations. 


\section{EXTENSIONS AND CONCLUSIONS}

In this paper we introduced the deterministic WIRELESS NETWORK JAMMING PROBLEM and provided several formulations using node covering constraints as well as constraints on the connectivity indices of the network nodes. We also incorporated percentile constraints into the derived formulations. Further, we provided two case studies comparing the two formulations with and without the risk constraints.

With the introduction of this problem, we also recognize that several extensions can be made. For example, all of the formulations presented in this paper assume that the network topology of the enemy network is known. It is reasonable to assume that this is not always the case. In fact, there may be little or no a priori information about the network to be jammed. In this case, stochastic formulations should be considered and analyzed.

A generalization of the node coverage formulation including uncertainties in the number of communication nodes and their coordinates might be considered. For the connectivity index problem, there might exist uncertainties in the number of network nodes, their locations, and the probability that a node will recover a jammed link. Also, efficient heuristics such as Greedy Randomized Adaptive Search Procedure (GRASP) (Pitsoulis and Resende, 2002), Genetic Algorithms (Goldberg, 1989), and Tabu Search (Glover, 1986), should be designed so that larger real-world instances can be solved. These are only a few ideas and extensions that can be derived from this new and interesting combinatorial optimization problem.

\section{ACKNOWLEDGEMENTS}

The authors gratefully acknowledge the Air Force Office of Scientific Research for providing funding under project FA9550-05-1-0137. Finally, we are thankful to the editor and an anonymous reviewer whose comments and suggestions greatly improved the quality of this paper.

\section{REFERENCES}

Garey, M.R. and D.S. Johnson. 1979. Computers and Intractability: A Guide to the Theory of NP-Completeness. W.H. Freeman and Company.

Glover, F. 1986. "Future paths for integer programming and links to artificial intelligence." Computers and Operations Research 5:533-549.

Goldberg, D.E. 1989. Genetic Algorithms in Search, Optimization, and Machine Learning. Addison-Wesley.

Holton, G. 2003. Value-at-Risk: Theory and Practice. Academic Press.

King, J. 1994. "Three problems in search of a measure.” American Mathematical Monthly 101:609-628.

Krokhmal, P., J. Palmquist and S. Uryasev. 2002. "Portfolio optimization with conditional value-at-risk objective and constraints." The Journal of Risk 4(2):11-27.

Krokhmal, P., R. Murphey, P. Pardalos, S. Uryasev and G. Zrazhevski. 2003. Robust decision making: Addressing uncertainties in distributions. In Cooperative Control: Models, Applications, and Algorithms, ed. S. Butenko, R. Murphey and P. Pardalos. Kluwer Academic Publishers pp. 165-185.

Pitsoulis, L.S. and M.G.C. Resende. 2002. Greedy randomized adaptive search procedures. In Handbook of Applied Optimization, ed. M.G.C. Resende and P.M. Pardalos. Oxford University Press pp. 168-183.

Resende, M.G.C. and P.M. Pardalos. 2006. Handbook of Optimization in Telecommunications. Springer.

Rockafellar, R.T. and S. Uryasev. 2000. "Optimization of conditional value-at-risk." The Journal of Risk 2(3):2141.

Rockafellar, R.T. and S. Uryasev. 2002. "Conditional value-at-risk for general loss distributions." Journal of Banking and Finance 26:1443-1471.

Uryasev, S. 2000. "Conditional value-at-risk: Optimization algorithms and applications.” Financial Engineering News 14:1-5. 
(C.W. Commander) Air Force Research Laboratory, Munitions Directorate, and, Dept. OF INDUSTRIAL AND SYSTEMS ENGINEERING, UNIVERSITY OF FLORIDA, GAINESVILLE, FL USA.

E-mail address: clayton. commander@eglin.af.mil

(P.M. Pardalos) Center for ApPlied Optimization, Dept. of Industrial and Systems EngiNEERING, UNIVERSITY OF FLORIDA, GAINESVILLE, FL, USA.

E-mail address: pardalos@ufl.edu

(V. Ryabchenko, S. Uryasev, and G. Zrazhevsky) Risk Management and Financial EngiNEERING LAB, DEPT. OF INDUSTRIAL AND SYSTEMS ENGINEERING, UNIVERSITY OF FLORIDA, GAINESVILLE, FL USA.

E-mail address: $\{$ valeriy, uryasev\}@ufl.edu 